\section{Análise de custo-efetividade em relação às terapias renais substitutivas: como pensar estudos em relação a essas intervenções no Brasil?}

\author{
Cost-effectiveness analysis of renal replacement \\ therapies: how should we design research on these \\ interventions in Brazil?
}

\author{
${ }^{1}$ Secretaria de Estado da \\ Saúde de Minas Gerais, \\ Belo Horizonte, Brasil. \\ 2 Instituto de Medicina \\ Social, Universidade do \\ Estado do Rio de Janeiro, \\ Rio de Janeiro, Brasil. \\ Correspondência \\ L. G. Sancho \\ Secretaria de Estado da \\ Saúde de Minas Gerais. \\ Rua Sapucaí 429, 10 o andar \\ Belo Horizonte, $M G$ \\ 30150-050, Brasil. \\ lsancho@uol.com.br
}

\begin{abstract}
This study aims to contribute to the discussion on the possibility of applying health economics assessment, specifically the cost-effectiveness technique, to renal replacement therapies for end-stage renal failure. A review was conducted on the interventions and their alternative courses from the perspective of the various methodological proposals in the literature, considering the availability of data and information in Brazil to back this type of research.

Cost-Effectiveness Evaluation; Renal Replacement Therapy; Chronic Kidney Failure
\end{abstract}

Leyla Gomes Sancho 1

Sulamis Dain 2

\section{Introdução}

O grande volume de recursos financeiros do orçamento ambulatorial do Sistema Único de Saúde (SUS) alocado nos procedimentos relativos às terapias renais substitutivas, associado à perspectiva da tendência de um aumento exponencial desta alocação decorrente do aumento da expectativa de vida e, por conseguinte, do incremento da prevalência de patologias de base que por sua história natural resultam em insuficiência renal crônica terminal/falência renal crônica, configuram a utilização da avaliação econômica, particularmente a análise de custo-efetividade, como um importante aliado para subsidiar uma alocação de recursos de forma exeqüível, eficiente e tendo a eqüidade como uma dimensão relevante a ser considerada.

O grande dilema, no entanto, é como viabilizar avaliações econômicas no nosso meio. Não só pela inexistência de uma padronização metodológica para a sua operacionalização, como também pelo seu alto custo e pela disponibilidade de dados e informações necessárias.

Nessa medida, este estudo pretendeu avaliar as possibilidades para a elaboração de estudos, por meio da análise de custo-efetividade no que tange as terapias renais substitutivas, e de acordo com os achados advindos desta avaliação, sugerir a mais factível. 


\section{Metodologia}

Trata-se de um estudo teórico que, direcionado por metodologias já consagradas na literatura internacional, avaliou e analisou as possibilidades factíveis à nossa realidade para a consecução de estudos desta ordem, avaliando os dados e informações sobre as terapias renais substitutivas disponíveis no nosso meio, métodos e proposições metodológicas referidas na literatura.

A avaliação sobre a possível construção de um estudo de análise de custo-efetividade sobre as terapias renais substitutivas, no que se refere aos dados e informações relativas e necessárias para esta construção, foi direcionada a partir do disposto no checklist elaborado por Drummond et al. 1 e no guia proposto por Gold et al. 2, que sistematizam os pontos de interesse para a execução de um estudo de análise de custo-efetividade.

Com base em uma revisão bibliográfica sobre proposições metodológicas utilizadas por distintas instituições e/ou organizações em alguns países, se ponderou estas proposições com a disponibilidade dos dados e informações sobre o objeto de interesse, para então prescrever uma possibilidade factível para a finalidade da discussão, modelar um estudo de custo-efetividade em relação às terapias renais substitutivas em nosso meio.

\section{Resultados}

À guisa das metodologias referidas como suporte para este estudo, o primeiro passo para a operacionalização de uma análise de custo-efetividade é a problematização, que engloba a descrição da doença em termos clínicos, epidemiológicos e o impacto destes em relação às questões sociais, políticas e econômicas, e a descrição das alternativas de intervenção sob comparação no que tange os benefícios e riscos e destes em termos econômicos.

\section{A descrição da doença em termos clínicos}

A descrição, em vista da história natural da doença e de sua evolução prognóstica, traz subsídios (informações) importantes para a estruturação do modelo de decisão a ser adotado na análise de custo-efetividade. Ou seja, o reconhecimento das causas de falência renal crônica na nossa realidade e os determinantes em relação à evolução prognóstica direcionam o planejamento de ações clínicas e a adoção de estratégias de intervenção que serão consideradas ao se construir o algoritmo do modelo de decisão.
No caso da falência renal crônica, que advém de vários grupos sindrômicos ou patologias de base, entre elas, o diabetes e a hipertensão enquanto fatores preditivos, surge quando o paciente tem apenas $15 \%$ de funcionalidade renal.

Os fatores de risco para a doença, segundo a Sociedade Brasileira de Nefrologia (SBN; http:// www.sbn.org.br), são subdivididos em diferentes grupos: (1) fatores de susceptibilidade: aumentada para a lesão renal (tais como: história familiar, obesidade e idade); (2) fatores desencadeantes: os que diretamente desencadeiam a lesão renal (tais como: a diabetes, a hipertensão, as infecções do trato urinário, as doenças auto-imunes e o efeito tóxico de drogas); (3) fatores de progressão: declínio da função renal, tais como o nível alto de proteinúria, controle precário da hipertensão e do diabetes; (4) fatores de doença avançada: morbidade e mortalidade aumentadas devido ao encaminhamento tardio, ao acesso vascular temporário, à albumina sérica baixa, anemia e dose mais baixa de diálise (medido pelo Kt/V).

A SBN, baseando-se em diretrizes traçadas pela National Kidney Foundation (NKF; http:// kidney.org/), também adota uma classificação para os estágios de insuficiência renal crônica associada a diferenças nas taxas de função glomerular (depuração do plasma pelo rim). Os estágios de interesse para o caso da falência renal crônica e relativo às terapias renais substitutivas são: o estágio 4: taxa de função glomerular (ml/ $\min / 1,73 \mathrm{~m}^{2}$ ) entre 15-29 (grave diminuição), ação de preparação para a terapia renal de substituição e; o estágio 5: taxa de função glomerular $\left(\mathrm{ml} / \mathrm{min} / 1,73 \mathrm{~m}^{2}\right) \leq 15$ (falência renal), ação de substituição.

No Brasil, dependendo da fonte de dados e dos estudos empíricos realizados, as causas mais freqüentes da insuficiência renal crônica estão dispostas na Tabela 1 .

Depreende-se desse quadro que os valores, tanto os advindos da base de dados referente aos pagamentos realizados pelo SUS 3 , quanto o do censo da SBN 4, demonstram uma dissensão. A qual, justaposta ao percentual referente à causa desconhecida ( $28 \%$ - SUS e $16 \%$ - SBN), evidencia uma primeira dificuldade para que esses dados subsidiem, entre outros elementos, o cálculo de parâmetros e as probabilidades de transição da modelagem de um estudo de análise de custoefetividade.

\section{A descrição da doença em termos epidemiológicos}

A descrição, em grande medida, subsidia a avaliação da efetividade das intervenções, o conhecimento das necessidades de atenção e a deman- 
da por serviços específicos, que, por conseguinte, indicam uma previsão para a oferta necessária. Esta, em última análise, é um indicativo para a escolha das alternativas a serem comparadas, bem como serve de referência para a previsão de uma distribuição eqüitativa da atenção/cuidado dos serviços.

A mensuração da prevalência e incidência em relação à falência renal crônica é dada no Brasil com base em instrumentos de pagamentos 5 . Diferentemente da forma como é medida nos Estados Unidos, por coleta mediante registros de pacientes.

No Brasil, nos últimos anos, foram poucos os estudos que trataram desse tema sob esse enfoque: Estudo Epidemiológico Brasileiro sobre Terapia Renal Substitutiva 3; Censo SBN 2002: Informações Epidemiológicas das Unidades de Diálise do Brasil 6; Epidemiologia da Doença Renal Crônica no Brasil 2004: Cenário Atual 7; e Inquérito Epidemiológico em Unidades de Diálise do Brasil 8. Ressalta-se que não se pesquisou as bases de dados referentes a dissertações e teses de instituições educacionais.

Estudo realizado no ano de 2002, pelo Monte Tabor Hospital São Rafael, encontrou uma incidência de $1.427 \pm 136$ (desvio-padrão) pacientes/mês em relação à média, e de 1.424 pacientes/mês em relação à mediana, tendo ocorrido aumento de 6,7\% de casos novos entre os anos de 1998 e 1999 3 . A prevalência da doença renal em terapias renais substitutivas no período de abril de 1997 a julho de 2000 foi de 524 por milhão de habitantes (/pmp). A prevalência pontual, ou seja, aquela estimada como a proporção de indivíduos de uma população de estudo de tamanho $\mathrm{N}$ que apresente a doença de interesse no instante $\mathrm{T} 9$, foi de 323/pmp em 2000.

Dados recentes evidenciam que o aumento médio na prevalência entre os anos de 2000 a 2002 foi de $6 \%$, ou seja, uma prevalência no ano de 2002 de 306/pmp, à luz dos dados do SUS, ou de $321 /$ pmp, contabilizados os $5 \%$ de pacientes em diálise em medicinas de grupos e/ou seguros de saúde particulares ${ }^{3}$. Adicionando os pacientes com enxerto renal funcionante, o valor se eleva para 409/pmp a 439/pmp. Segundo dados da SBN 6, a prevalência no ano de 2002 era de 54.523 pacientes em tratamento que, em 2003/2004, se eleva para 559.153 pacientes, ou 333/pmp.

Os dados advindos dos estudos acima referidos 3,6 evidenciam que o Brasil tem o quarto maior número de pacientes mantidos em diálise e apresenta uma taxa de prevalência quatro vezes menor do que os Estados Unidos 2 e bem abaixo de vários países desenvolvidos 5 .

A Tabela 2 dispõe os dados de incidência e prevalência de diálise na perspectiva interna-
Tabela 1

Causas de insuficiência renal crônica com base na Sociedade Brasileira de Nefrologia (SBN) e no Sistema Único de Saúde (SUS)

\begin{tabular}{lcccc}
\hline Causa & \multicolumn{2}{c}{ SBN } & \multicolumn{2}{c}{ SUS } \\
& $\mathbf{n}$ & $\%$ & $\mathbf{n}$ & $\%$ \\
\hline Glomerulonefrite & 666 & 23,5 & - & - \\
Glomerulopatias & - & - & 16.841 & 18,9 \\
Hipertensão & 684 & 24,1 & 20.990 & 23,6 \\
Diabetes tipo 1 & 71 & 2,5 & 12.266 & 13,8 \\
Diabetes tipo 2 & 399 & 14,1 & - & - \\
Nefrite intersticial & 81 & 2,9 & - & - \\
Rins policísticos & 78 & 2,7 & - & - \\
Uropatia obstrutiva & 86 & 3,0 & - & 9,9 \\
Outros & 321 & 11,3 & 8.797 & 28,0 \\
Desconhecida & 453 & 16,0 & 24.865 & 5,8 \\
Indeterminado & - & - & 5.122 & 100,0 \\
Total & 2.839 & 100,0 & 88.881 & \\
\hline
\end{tabular}

Fonte: com base na SBN (Sesso 31); de acordo com o SUS (Ministério da Saúde 3).

cional comparada ao Brasil. Nosso país, conforme esperado, não apresenta de forma sistemática dados relativos à incidência ou prevalência, apenas por meio de estudos empíricos realizados.

Um bom indicador para a avaliação das terapias renais substitutivas é a mortalidade, expressa pela sobrevida (dependente da idade e da co-morbidade) em diferentes tempos do tratamento, e a morbidade pela taxa de complicações, em geral expressa pela hospitalização. Se o objetivo é avaliar a qualidade da atenção (processo), utilizam-se também como indicadores o número de episódios mórbidos agudos ocorridos durante as sessões e a taxa de soroconversão para hepatite 10 .

No Brasil, segundo o Estudo Epidemiológico Brasileiro sobre Terapia Renal Substitutiva 3, o percentual de óbitos dos pacientes em diálise no período estudado foi de 28,9. Destes, $26 \%$ ocorreram num intervalo menor do que 2 meses de Autorização de Pagamento de Alto Custo (APAC); $19,7 \%$ de 6 a 12 meses; 18,7\% com 12 meses e $35,6 \%$ entre 2 e 6 meses. A medida da mortalidade do estudo em questão não considerou o conceito usual de taxa de mortalidade, em que o denominador da razão é a população geral. No caso, o denominador é composto pelo número de pacientes/ano em terapia renal substitutiva. Os pacientes no Brasil só iniciam o tratamento após a liberação da APAC.

De acordo com o exposto, novamente se depara com o problema já evidenciado, o da insuficiência e não coincidência de dados que sub- 
Tabela 2

Incidência e prevalência de insuficiência renal crônica em diversos países (por milhão de habitantes residentes) em 2001 e 2002

\begin{tabular}{lcccc}
\hline País & \multicolumn{2}{c}{ Ano } & \multicolumn{2}{c}{2002} \\
& Incidência & Prevalência & Incidência & Prevalência \\
\hline Brasil & - & - & - & 321 \\
Japão & 252 & 1.642 & 254 & 1.726 \\
Estados Unidos & 328 & 1.403 & 336 & 1.446 \\
Alemanha & 184 & 921 & 174 & 920 \\
Portugal & 199 & 918 & - & - \\
Dinamarca & 138 & 679 & 129 & 711 \\
Suécia & 126 & 735 & 124 & 756 \\
Holanda & 101 & 621 & 100 & 658 \\
Espanha & 128 & 885 & 92 & 888 \\
Inglaterra & 93 & 566 & - & - \\
\hline
\end{tabular}

Fonte: United States Renal Data System 42; European Renal Association/European Dialysis and Transplant Association 43 sidiam a construção de um estudo de análise de custo-efetividade.

\section{A descrição das alternativas da intervenção sob comparação}

Antes mesmo da descrição sobre quais alternativas serão comparadas, é necessário fazer uma avaliação preliminar das opções de tratamento existentes - quais as mais pertinentes em vista da sua efetividade, segundo a nosologia pré-dialítica prevalente em nosso meio e a conseqüente indicação terapêutica, assim como sua distribuição de acordo com o status sócio-econômico da população usuária das terapias renais substitutivas, dado que as diversas modalidades e seus espaços de atenção (settings) requerem uma série de condições específicas, nem sempre acessíveis a determinados grupos populacionais.

No nosso país são dois os tipos de diálise: a peritoneal e a hemodiálise. A peritoneal se apresenta em três diferentes modalidades: a intermitente (DPI), a ambulatorial contínua (CAPD) e a automática (DPA); e a hemodiálise padrão (standard/status quo), dispensada três vezes por semana durante quatro horas/dia, em diferentes espaços (settings), hospitalar/centros satélites hospitalares e em unidades independentes/centros de referência.

Ressalta-se também a hemodiálise no domicílio, implementada no Brasil, porém não contemplada pelo SUS, e a conhecida como short duration daily dialysis (DHD), dispensada seis vezes por semana durante duas horas/dia e oferecida à população por alguns planos de saúde e realizada pelas clínicas atreladas à Fresenius (multinacional do ramo de máquinas dialisadoras), que segundo estudo prospectivo realizado no Brasil, durante dois anos por Lugon et al. 11, concluiu que há melhora significativa em alguns sintomas, tais como: diminuição, em até dez vezes, de crises hipertensivas, episódios de hipotensão, dores de dente e vômitos, assim como declínio nos níveis de uréia e creatinina pré-dialítica e aumento no valor médio do hematócrito. Outro estudo, realizado no Brasil por Salomão et al., 12 corrobora os achados de Lugon et al. $11 \mathrm{e}$ salienta a melhora no valor do Kt/V semanal (de $3,95 \pm 0,47$ para $6,65 \pm 1,16$ ).

No Brasil, por intermédio do SUS, estão disponíveis outras tecnologias referentes à fase prérenal. Para o SUS, tais ações se consubstanciam na criação dos Centros de Referência em Nefrologia, os quais têm como competência prestar uma assistência integral e integrada aos portadores de doenças renais.

A distribuição dos pacientes em nosso meio por modalidade se inscreve conforme a Tabela 3 .

A Tabela 4, como complemento à acima referida, mostra o número de APACs para transplantes entre abril de 1997 e julho de 2000, que, se relativizado com outros tipos de diálise, representa apenas $2,9 \%$ do total.

No tocante à relação entre a modalidade da intervenção e a indicação terapêutica, a qualidade de vida do paciente é a variável descrita como a de maior importância. Inquérito elaborado entre nefrologistas britânicos e irlandeses, numa escala de 1-5, refere que a preferência do paciente $(4,4)$ é o fator preponderante para a indicação referente à modalidade de diálise, seguido pela qualidade de vida $(3,8)$, pela morbi-mortalidade $(3,6)$ e o custo, seja para o paciente e/ou para o sistema, como o menos importante 13 . Afirmativa que é contradita e referida na literatura, dado que a escolha com base no custo pode se dar inclusive pelos incentivos gerados pelos sistemas de pagamentos 14 .

À luz dos estudos internacionais de análise de custo-efetividade sobre terapias renais substitutivas, desde o primeiro, datado de 1968 15, o resultado é favorável ao transplante renal. E, diante dessa supremacia, o que atualmente se observa é a elaboração de estudos que realizam comparações entre as outras modalidades e/ou formas de hemodiálises (domiciliar, centros de cuidado e outras) e entre os diversos elementos (membranas) dos equipamentos, reuso de dialisador e mesmo de fármacos 16,17,18,19,20,21.

De qualquer sorte, e como uma premissa à consecução de estudos, se deve levar em consi- 
Pacientes mantidos em terapia de substituição renal no Brasil, 1999 a 2004.

\begin{tabular}{|c|c|c|c|c|c|c|c|c|c|c|}
\hline \multirow[t]{3}{*}{ Tratamento } & \multicolumn{10}{|c|}{ Ano } \\
\hline & \multicolumn{2}{|c|}{1999} & \multicolumn{2}{|c|}{2000} & \multicolumn{2}{|c|}{2001} & \multicolumn{2}{|c|}{2002} & \multicolumn{2}{|c|}{$2003 / 2004$} \\
\hline & $\mathbf{n}$ & $\%$ & $\mathbf{n}$ & $\%$ & $\mathbf{n}$ & $\%$ & n & $\%$ & $\mathbf{n}$ & $\%$ \\
\hline Hemodiálise & 38.221 & 89,5 & 41.900 & 90,0 & 43.700 & 89,5 & 48.874 & 89,6 & 53.139 & 89,83 \\
\hline Diálise peritoneal & 4.474 & - & 4.657 & - & 5.106 & - & 5.649 & & 6.014 & - \\
\hline DPAC & 3.625 & 8,5 & 3.587 & 7,7 & 3.667 & 7,5 & 3.728 & 6,8 & 3.776 & 6,38 \\
\hline DPA & 195 & 0,5 & 538 & 1,2 & 1.030 & 2,1 & 1.570 & 2,9 & 1.963 & 3,32 \\
\hline DPI & 654 & 1,5 & 532 & 1,1 & 409 & 0,9 & 351 & 0,7 & 275 & 0,47 \\
\hline Total em diálise & 42.695 & 100,0 & 46.557 & 100,0 & 48.806 & 100,0 & 54.523 & 100,0 & 59.153 & 100,0 \\
\hline
\end{tabular}

DPAC: diálise peritoneal ambulatorial contínua; DPA: diálise peritoneal automática domiciliar; DPI: diálise peritoneal intermitente.

Fonte: Romão Jr. et al. 6 e Sociedade Brasileira de Nefrologia 44.

Tabela 4

Informações sobre o transplante renal no Brasil.

\begin{tabular}{lllllll}
\hline & $\mathbf{N}$ & $\%$ & & Masculino & Média de idade Desvio-padrão \\
\hline Transplante & 2.544 & 2,9 & 1.505 & 59,8 & 39,6 & 13,1 \\
\hline
\end{tabular}

Fonte: Ministério da Saúde 3.

deração a disponibilidade (formas e modalidades das diálises) à população e a acessibilidade desta mesma população em relação às alternativas sob comparação; mesmo que a mais custoefetiva traga, como conseqüência, uma mudança na sua utilização/disponibilidade.

\section{Evidências empíricas sobre o problema no Brasil}

Nesta etapa de um estudo sobre custo-efetividade, a pretensão com base em estudos empíricos e dos dados e informações disponíveis, é situar o problema à luz de um determinado contexto. Assim como, considerar o impacto do problema e das intervenções sob análise em termos econômicos.

No Brasil, face à perspectiva de se garantir uma melhora no estado de saúde da população sob falência renal e diante da restrição orçamentária, há evidente lacuna em relação a estudos de avaliação econômica (custo-efetividade/utilidade) sobre terapias renais substitutivas, principalmente em relação àquelas que geram importantes ganhos em saúde/benefícios, vis-à-vis seu custo.
No Brasil existe um único estudo indexado sobre a análise de custo-efetividade em relação ao tratamento da falência renal crônica. O estudo, elaborado por Sesso et al. 22 , concluiu, após dois anos de pesquisa e comparações feitas entre os quatro tratamentos, que o custo por ano de sobrevida era de US\$12.134,00 para o CAPD, de US\$ 10.065,00 para a hemodiálise hospitalar, de US $\$ 6.978,00$ para o transplante renal (doador cadáver) e de US\$ 3.022,00 (doador vivo).

Os gastos realizados pelo Ministério da Saúde em relação aos procedimentos relacionados às terapias renais substitutivas, no ano de 2003 e computados até o mês de outubro, foram de aproximadamente R\$ 821.515.748,00 que, se comparados aos valores do mesmo mês do ano anterior (R\$ 431.767.956,00), refletem um acréscimo próximo de $54 \%$, percentual que indubitavelmente não é superado por nenhuma correção anual dos índices econômicos 23 . O acréscimo justifica um real aumento de aporte de recursos para os procedimentos da terapia renal substitutiva, os quais podem ser explicados pela absorção da demanda devido ao aumento da oferta para o tratamento da insuficiência renal crônica; em vista, inclusive, do aumento real dos valores pagos $(10 \%)$ para os procedimentos relativos às 
terapias renais substitutivas através da tabela do Sistema de Informação Ambulatorial (SIA) do SUS 23 .

$\mathrm{O}$ atendimento ao paciente em falência renal crônica não se limita às sessões de hemodiálise, pois inclui também a garantia de outros procedimentos como a dispensação de medicamentos, o acesso à internação hospitalar, quando necessária, e o acesso ao transplante renal. Assim sendo, o gasto total do SUS, no ano, foi de 1,2 bilhão de Reais, com 50 mil pacientes 23 . Os gastos com terapias renais substitutivas, se comparados aos gastos totais executados pelo Ministério da Saúde no ano de 2003 (R\$ 17.414.742.205,28) 24, são de aproximadamente $8 \%$, o que demonstra sua efetiva relevância. Ressalta-se que não é possível valorar com precisão o dado porque os números apresentados em diversas notas oficiais, advindos da própria página eletrônica Ministério da Saúde, referem valores distintos, que variam de $93 \%$ a $98 \%$.

Segundo os dados Departamento de Informática do SUS (DATASUS) 24, órgão da Secretaria Executiva do Ministério da Saúde, os gastos apenas com as terapias renais substitutivas foram de $\mathrm{R} \$ 233.255 .181,79$ em 2003 e R\$ 686.200.400,97 em 2004

Em termos macroeconômicos, segundo Romão et al., 6 há uma relação direta entre a prevalência, o número de centros de diálise e o Produto Interno Bruto (PIB) regional - onde o PIB é maior há maior acesso.

O que se depreende então dessa situação, independentemente da importância das terapias renais substitutivas à luz da efetividade de cada intervenção ou curso alternativo da intervenção ou da alocação de recursos pelo governo, é que não temos no nosso meio o suporte suficiente em termos de informação e/ou evidência científica para, com a menor margem de segurança, executar um estudo de avaliação econômica sobre as terapias renais substitutivas sem que se incorra em algum viés.

\section{Avaliação da efetividade}

Outro ponto a ser avaliado é o da efetividade das alternativas escolhidas para a comparação.

A efetividade clínica tem como objetivo primário avaliar determinada tecnologia em termos de benefício e dano e, secundariamente, ao ser justaposto ao custo, avaliar a eficiência (análise econômica) de uma tecnologia. No caso das terapias renais substitutivas, à luz da falência renal, os estudos são, em geral, do tipo retrospectivo ou prospectivo-observacionais 25 .

Revisão sistemática realizada por Mowatt et al. 26 sobre algumas alternativas terapêuticas re- lativas às terapias renais substitutivas pontua, no tópico sobre efetividade, aspectos de interesse encontrados nos estudos sob avaliação. Os tipos de estudos mais comuns foram o observacional e a revisão sistemática, e a quase totalidade contempla os dados sócio-econômicos e as co-morbidades. As medidas utilizadas foram o quality of life (QoL), a taxa de hospitalização, o status de escolaridade e de emprego, o fracasso da técnica e do acesso, a anemia, o uso da eritropoietina, os índices bioquímicos, o Kt/V, a pressão arterial e mortalidade/sobrevivência.

Os estudos nessa revisão que utilizaram a sobrevivência como resultado optaram, como método estatístico, o modelo de Cox, cuja técnica de regressão ajusta as características de base dos diferentes subgrupos. O modelo provê um resultado a partir de uma razão de risco que é comparado com um valor de referência. As variáveis mais utilizadas nesse modelo foram: a idade de início do tratamento, a presença de diabetes, as causas de end stage renal disease (ESRD) e as comorbidades (bronquite crônica, artrite, doenças cardiovascular e vascular periférica).

Um ponto de inflexão para um estudo sobre terapias renais substitutivas é a avaliação da eficácia/efetividade das alternativas. Partindo-se do pressuposto de que, para nosso meio, diferentemente de países desenvolvidos, a existência de um benefício marginal em termos comparativos de efetividade não é suficiente para uma tomada de decisão - assertiva fundamentada nos estudos de Silva 27,28,29. Outros aspectos devem ser levados em consideração, como por exemplo, a operacionalização da tecnologia em determinado contexto.

Estudo realizado por Sesso et al. 22 em relação a pacientes de nosso meio, optou pela sobrevida como medida de resultados para a efetividade. Para esta análise, retrospectiva, a data $\left(\mathrm{T}_{0}\right)$ foi a do início do tratamento, e a do tempo final coincidiu com a do término do estudo (dois anos). A avaliação foi realizada no final do primeiro ano de acompanhamento (follow-up) e do segundo. $\mathrm{O}$ resultado de interesse foi que, no primeiro ano, a hemodiálise apresentou uma sobrevida de $100 \%$; a do CAPD, $90 \%$; a do transplante de relativos vivos, $92 \%$; e a do transplante com cadáver, $70 \%$. No segundo ano, a taxa acumulada foi: hemodiálise, 91\%; CAPD, 90\%; transplante de relativos vivos, $92 \%$; e transplante com cadáver, $70 \%$. Ao ajustar o valor pela qualidade de vida houve inversão, demonstrando ser o transplante mais efetivo.

Estudo de Carvalho et al. 30 que avaliou a sobrevida no Estado do Rio de Janeiro, Brasil, de 1998 a 2001, e entre os centros de diálise utilizando o modelo de riscos proporcionais de Cox, 
concluiu que as unidades de diálises de maior porte apresentam pacientes com sobrevida maior do que a das de menor porte. Esse resultado, se comparado ao estudo de Sesso ${ }^{31}$, indica que a sobrevida não tem só uma relação com o tipo de terapia renal substitutiva, mas também no que tange o espaço (setting). Esse fato respalda a noção de que a experiência no manejo de pacientes é também um dado importante a ser considerado em uma avaliação econômica sobre as terapias renais substitutivas que, em síntese, está relacionada com a utilização do serviço.

Diante da característica da insuficiência renal crônica em sua fase terminal, e independentemente das propostas de alternativas a serem comparadas, a medida ideal de resultado para avaliar a efetividade é a sobrevida. Esta, entretanto, já na perspectiva da análise do custo-efetividade, deve ser ajustada pela qualidade de vida.

No Brasil já existem alguns questionários validados para o caso específico da insuficiência renal crônica, tais como: o Kidney Disease Quality of Life Short Form (KDQOL-SF) e o Medical Outcomes Study 36 Item Short - Form Health Survey (SF-36), validados por Ciconelli et al. 32 e utilizados na ESRD por Rodrigues-Neto et al. 33 .

Outra alternativa para uma avaliação da efetividade das terapias renais substitutivas, principalmente aquela em que a hemodiálise esteja contemplada, é por meio de medidas intermediárias relevantes para nosso contexto. Nesse caso, dois aspectos devem ser relativizados: o clínico e o econômico. Na especificidade do clínico, a anemia e o Kt/V são as medidas de escolha; na especificidade do econômico, a eritropoietina e a admissão hospitalar impactam a alocação de recursos. E todas têm a sobrevida com end-point.

Em relação às diálises peritoneais, medida de interesse tanto no aspecto clínico como no econômico, são a infecção e a admissão hospitalar. No que tange o transplante, a medida de escolha é a sobrevida ajustada pela qualidade de vida.

Diante do cenário apresentado, do número insuficiente de estudos que avaliem a efetividade das terapias renais no nosso meio e em face da inexistência de dados para o caso brasileiro, principalmente os que se referem à efetividade à luz de dados demográficos e sócio-econômicos, a possível solução para esta análise, dependendo do enfoque, é uma estimativa local.

\section{Avaliação do custo}

Outro item de interesse em estudos de análise de custo-efetividade é a identificação, mensuração e valoração dos recursos consumidos que compõem a função de produção das intervenções ou distintos cursos de ação de uma intervenção sob comparação.

Conforme citado, o único trabalho sobre análise de custo-efetividade realizado no Brasil foi o de Sesso et al. 22. De interesse para esta discussão, chama a atenção o fato de que os valores em relação aos diversos itens e, por conseguinte, do somatório da parcela relativa ao custo, têm relação direta com os baixos salários pagos ao staffe a reutilização de cateteres.

Em meados de 1997, a SBN, a Associação Brasileira de Centros de Diálise e Transplantes (ABCDT) e a seção regional do Estado de São Paulo da SBN solicitaram à Fundação Instituto de Pesquisas Econômicas (FIPE) estudo sobre a estrutura de custos dos serviços de hemodiálise em São Paulo, Brasil 34. O objetivo do estudo foi avaliar o impacto dos custos operacionais nos diversos centros de diálise, a partir da implementação da Portaria $n^{\circ}$. 38, de 3 de março de 1994, que regulamenta a técnica para o funcionamento dos serviços de terapia renal substitutiva e normatiza o cadastramento destes centros junto ao SUS, e antes e após a da Portaria $n^{\circ}$. 2.042, de 11 de outubro de 1996, do Ministério da Saúde 34 . Não é nosso objetivo fazer juízo de valor a respeito da metodologia ou mesmo do resultado do estudo da FIPE, mas evidenciar algumas inferências importantes abordadas pelos autores do estudo.

A primeira questão, que não causa surpresa, é o fato de que há diferença de valores de acordo com a economia de escala. Outra questão pontuada é a diferença de valores (custos de produção) entre os diferentes serviços que prestam atenção ao portador de falência renal - o público é menos oneroso e o privado tem maior custo, sendo o filantrópico de valor intermediário. Essa diferença, segundo a FIPE 34, sob uma perspectiva, se deve aos encargos e taxas/impostos imputados ao serviço privado, dos quais o setor público e o serviço filantrópico estão isentos e, em outra perspectiva, devido ao fato de que os hospitais privados e unidades autônomas compartilham instalações e serviços com outras atividades, ou seja, há um incremento de custo devido ao overhead (custos administrativos). Os valores encontrados estão dispostos na Tabela 5, ressaltando-se que o valor pago à época pelo SUS para o procedimento (sessão de hemodiálise) era de R\$ 89,12.

Outro estudo realizado recentemente pelo Instituto de Saúde Coletiva da Universidade Federal da Bahia 35, encontrou como resultado para o custo de produção valores diferentes para as sessões de hemodiálise de acordo com o porte do estabelecimento (Tabela 6), ressaltando-se que o valor do procedimento no período era de $\mathrm{R} \$ 108,00$. 
Tabela 5

Valores da sessão de hemodiálise em Reais ( $R$ \$).

\begin{tabular}{lcc}
\hline & $\begin{array}{c}\text { Custo anterior à } \\
\text { Portaria } \mathbf{n}^{\circ} . \mathbf{2 . 0 4 2}\end{array}$ & $\begin{array}{c}\text { Custo posterior à } \\
\text { Portaria } \mathbf{n}^{\circ} \mathbf{. 2 . 0 4 2}\end{array}$ \\
\hline Médio & 94,79 & 167,02 \\
Unidades autônomas & 127,73 & 167,02 \\
Unidade de diálise hospitalar & 123,85 & 153,70 \\
Filantrópicas & 100,97 & 123,70 \\
Pública & 94,79 & 114,30
\end{tabular}

Fonte: Fundação Instituto de Pesquisas Econômicas 34.

Tabela 6

Custo da sessão de hemodiálise em Reais (R\$), em clínicas e unidades hospitalares.

\begin{tabular}{lcccccc}
\hline & & Clínicas & \multicolumn{3}{c}{ Unidades hospitalares } \\
Mês & A & B & Média & C & D & Média \\
\hline Setembro & 95,23 & 94,72 & 94,97 & 125,49 & 142,25 & 133,87 \\
Outubro & 88,63 & 91,21 & 89,92 & 114,47 & 134,52 & 124,49 \\
Novembro & 95,22 & 98,80 & 97,01 & 151,87 & 153,15 & 152,51 \\
\hline
\end{tabular}

Fonte: Instituto de Saúde Coletiva, Universidade Federal da Bahia 35.

A ABCDT disponibiliza, em sua página eletrônica (http://www.abcdt.org.br), uma planilha de custos referente ao ano de 2003 que apresenta um terceiro grupo de valores, se comparado com os estudos anteriores.

É interessante observar que os valores encontrados nos três trabalhos referenciados tendem, ajustados para um mesmo período, a apresentar variabilidade. Com base no conteúdo das planilhas, o que se depreende é que os itens identificados, mensurados e valorados não são os mesmos entre os três estudos e que, diante de estudos realizados em diferentes contextos (regionais), estes podem apresentar diferentes valores/preços para os recursos consumidos.

A referência a esses estudos reflete exatamente o que é relatado na literatura, ou seja, a variabilidade entre os resultados dificultando a generalisability dos estudos de análise de custoefetividade 25,26,36.

A necessidade de consistência e padronização de métodos para avaliação econômica, particularmente no que se refere ao componente do custo, é um fato de reconhecida legitimidade. No entanto, ainda é visível uma diversidade entre os estudos, apesar da formalização de inúmeros guias metodológicos (guidelines).
Nesta perspectiva, e não diferente do encontrado no pífio número de estudos microeconômicos realizados no setor saúde, Adam et al. 37 , revisando estudos com o objetivo de avaliar as possíveis fontes de variação no que concerne aos métodos de apropriação de custos, concluíram que a maior fonte estava relacionada aos próprios guidelines. Os autores identificaram especificamente quatro situações: não há concordância entre os guidelines no que tange as recomendações; os guidelines não especificam como viabilizar as recomendações; as questões particulares quanto à metodologia não são discutidas; e há falta de aquiescência entre os guidelines.

\section{O modelo de decisão}

A etapa considerada chave para a elaboração de um estudo de avaliação econômica em saúde é a escolha da modelagem para a decisão, a qual depende do objetivo do estudo, do tipo de fonte de dados, e que representa a síntese das informações referentes à efetividade e custo; principalmente o impacto destas informações para o longo prazo e para a extrapolação de resultados em distintos subgrupos da população.

Para tanto, se pressupõe inicialmente que as questões no que concerne à fundamentação teórica, ou melhor, a escolha da abordagem e as controvérsias metodológicas, propositalmente não discutidas nesta publicação, sejam levadas em consideração, assim como se considerem quatro etapas para a consecução de uma modelagem, ou seja, a revisão sistemática dos dados relevantes, a estimação de todas as entradas (inputs) possíveis, a análise de sensibilidade e a validação do modelo.

Relevante estudo realizado por Kirby \& Vale 38 , dois reconhecidos pesquisadores nesta área do conhecimento, especificamente em temas relacionados à nefrologia, utilizou uma revisão sistemática para obter a probabilidade de transição entre os estados de saúde (modalidades de terapia renal substitutiva, de complicação e morte) e adotou o modelo de Markov para a avaliação econômica, representando os padrões de tratamento esperado. Os parâmetros utilizados no estudo foram: curva de sobrevivência de Kaplan-Meier e probabilidade quanto às complicações (organizadas em uma matriz).

$\mathrm{Na}$ perspectiva de um estudo que tem como objetivo o de direcionar a alocação de recursos é sempre necessário a escolha de um critério de decisão. No nosso meio, dada a lógica do financiamento do setor, ou seja, a do pagamento por procedimentos, não há definição $a$ priori de critérios de decisão, como, por exemplo, o limiar (adoção de limite orçamentário). Ao contrário, a 
lógica vigente do financiamento tende a incentivar a criação da demanda induzida pela oferta que, como conseqüência, produz interesse em captar o doente na fase final da doença, o que eleva inclusive o valor global da alocação (limiar) em termos de orçamento.

No que tange as técnicas de modelagem para a decisão, a maneira mais simples de consecução é a utilização da árvore de decisão (decision tree) relativa a um problema que apresenta diversas alternativas ou cursos alternativos.

Para a construção da árvore deve-se inicialmente identificar e limitar o problema; estruturar o problema ao longo do tempo; caracterizar as informações necessárias para preencher a estrutura de decisão e escolher o curso de ação baseado no resultado da estrutura de decisão. As árvores de decisão são construídas com base nas evidências clínicas (efetividade) e de dados secundários (custos), nas quais se justapõem nódulos de escolha (ponto na seqüência temporal em que há a seleção das alternativas ou cursos alternativos) e nódulos de chance (pontos seqüenciais temporais nos quais o evento é evidenciado).

A análise do resultado - incremental costeffectiveness ratio (ICER) - e a conseqüente análise de sensibilidade, em vista dos parâmetros utilizados para as estimativas, é normalmente avaliada por métodos estatísticos determinísticos, adequados para dados de fontes secundárias ou por métodos estocásticos para dados de fonte primária; ou ainda pelos métodos determinísticos-estocásticos, cujo efeito (efetividade) é medido e o custo estimado, e somente se avalia a variação dos efeitos.

Outra alternativa, mais complexa, porém em conformidade com a história natural da doença, principalmente as doenças crônicas que apresentam recorrências, ou com as mudanças no que se refere à terapêutica, é o uso do modelo de Markov que, sendo dinâmico, tem como vantagem a utilização das probabilidades de transição entre os distintos estados de saúde (estados de Markov) ao longo do tempo, e como desvantagem o fato de todos os pacientes em um estado de saúde serem configurados como idênticos e não comportar decisões em múltiplos períodos de tempo. Ou seja, o modelo simula o caminho percorrido pelo paciente através dos estados de saúde ao longo da evolução da doença.

A modelagem, dependendo do tipo de fonte de dados e da quantificação da incerteza em relação às estimativas dos parâmetros, pode ser determinística ou estocástica. O resultado da análise, incluído o teste de sensibilidade, e principalmente no caso deste ser conflitante e/ou insuficiente, pode ser avaliado a partir, por exemplo, de um teste de hipótese por meio de modelos
Bayesianos, e a incerteza através do intervalo de confiança (simulação de Monte Carlo). Vale destacar que o resultado também pode ser avaliado por métodos determinísticos ou estocásticos.

\section{Considerações}

Não foi objetivo deste estudo definir, $a$ priori, as alternativas a serem comparadas. Entretanto, os dados e informações expostos nesta discussão sugerem, em relação às diálises, a não consideração da diálise de curta duração e, já como um critério de exclusão, a hemodiálise domiciliar automatizada, não existente no Brasil, ou melhor, não disponibilizada à população pelo SUS.

A exclusão da diálise de curta duração, se avaliada na perspectiva econômica, tem como fundamento o fato de que certamente incorreria em diminuição de acesso, porque a relação entre o preparo do paciente e a diálise per si - a cada duas horas durante seis dias por semana - não tem correspondência proporcional, seja em termos de produtividade e, talvez, de custo, com relação ao preparo e diálise a cada quatro horas durante três dias por semana - o intervalo de giro se torna maior.

A hemodiálise domiciliar automatizada, não disponível para os pacientes do SUS, tem também como fator contra-indicativo, e mesmo impeditivo, o fato de requerer um tipo especial de atenção que apenas é facultado a uma pequena parcela da população.

Considerando como critério de inclusão para os estudos a configuração sócio-econômica dos pacientes em tratamento - dado não disponibilizado de forma sistematizada nos bancos oficiais - as diálises peritoneais (CAPD e DPA), pela necessidade de condições satisfatórias de higiene, podem ser fator contra-indicativo para determinados grupos sociais - os de baixa renda.

Ou seja, se o objetivo for avaliar as possibilidades para a população como um todo, e não especificamente para subgrupos populacionais - tendo a eqüidade como fator norteador da alocação de recursos -, as alternativas mais relevantes parecem ser o transplante renal (doador vivo ou cadáver) e a hemodiálise tradicional. Proposição que se justapõe ao estudo realizado por Sesso et al. 22, em 1990.

Outra opção, mais ousada, seria realizar um estudo entre a hemodiálise e o CAPD, caso se considerasse a lógica dos subgrupos populacionais, ou seja, se o CAPD fosse mais custo-efetivo para os de média e alta rendas e a hemodiálise para os de baixa renda. O resultado seria relevante, pois em certa medida aumentaria o acesso. 
Também como outra opção, sob a forma de screening, seria a Proposta de Atenção Integral ao Paciente Portador de Falência Renal. Ou ainda, como uma outra proposta para screening, sugerir a dosagem de microalbuminúria, que já demonstrou ser custo-benéfica 39.

Assim como já justificado, o grande desafio se atém à formalização e à consolidação de um sistema de informação capaz de subsidiar com os dados necessários a proposição das alternativas que deverão ser comparadas. E na especificidade dessa escolha é importante a conformação de uma base de dados que propicie a operacionalização de estudos empíricos confiáveis sobre a efetividade no que se refere às ações terapêuticas e para o (re)conhecimento da oferta, da demanda, da utilização e da distribuição da atenção.

Na perspectiva da avaliação de custos, não diferente do que foi discutido em relação à efetividade, um primeiro passo para a padronização metodológica é a estruturação de um sistema de informação em custos, de abrangência nacional, e este atrelado a um banco de dados regionais. Ou seja, a estruturação de uma base de dados atrelada, por exemplo, ao DATASUS.

Outra opção, caso a proposição inicial não seja factível, seria a realização de revisões sistemáticas e metanálise, com base em estudos elaborados em contextos similares ao nosso, adicionadas aos ajustes necessários (câmbio, inflação, desconto etc.). Ressalta-se que, como uma regra, é proposto pelos guidelines que os estudos sejam apresentados em dólares usando a taxa de câmbio Purchasing Power Parity (PPP). O PPP é o número de unidades que a moeda corrente local requer para comprar a mesma quantidade de uma unidade na moeda referenciada.

Outro passo que deve ser implementado para a realização de estudos de análise de custo-efetividade é a padronização em relação à tipologia dos custos indiretos e intangíveis a serem apropriados, ou melhor, a identificação dos custos relevantes, de acordo com o nosso contexto, conforme a possibilidade de obtenção de dados e destes na perspectiva temporal.

No que se refere à mensuração desses custos, uma opção é a utilização dos parâmetros referenciados pela Pesquisa Orçamentária Familiar/Instituto Brasileiro de Geografia e Estatística (POF/IBGE) e/ou de outros órgãos oficiais que trabalhem com dados desta ordem.

Entretanto, as possibilidades apresentadas dependem do tipo de desenho de estudo proposto. Os estudos de análise de custo-efetividade são em geral do tipo observacional (retrospectivo ou prospectivo) e, dependendo da amostra e das variáveis dependentes, os custos podem ser distintos. Ou seja, seria necessária a consecução de análises multivariadas ou de outra modelagem estatística para ajustar a variabilidade.

Face à escassez de estudos de avaliação/análise de custos em relação às diálises e ao transplante, uma proposta factível para as pesquisas a serem efetivadas, independentemente das alternativas sob comparação, no curto prazo, é a utilização dos valores das tabelas.

\section{Conclusão}

$\mathrm{Na}$ especificidade das terapias renais substitutivas, a existência de estudo prévio sobre custoefetividade apresenta-se como fator positivo, possibilitando a realização de um estudo, baseando-se nos dados do referido estudo, por meio da modelagem Bayesiana de Markov Chain Monte Carlo. Esta, já testada, pode ser associada ao emprego do programa WinBUG (MRC Biostatistics Unit, Cambridge, Reino Unido), já apropriado em nosso meio ${ }^{40}$. Essa opção para a consecução de um estudo de análise de custo-efetividade sobre as terapias renais substitutivas tem como fundamento o fato de já ter sido empregada por Spiegelhalter 41 , ou seja, ter apropriado estudo anteriormente publicado e aplicado o modelo.

De qualquer forma, ainda é muito limitada a quantidade de estudos que poderiam subsidiar com dados a estimativa de parâmetros, contribuindo para a elaboração de um estudo de avaliação econômica em saúde sobre as terapias renais substitutivas, tanto na perspectiva de avaliar outros cursos alternativos da intervenção, como em relação a outros espaços possíveis de dispensação (utilização) desta intervenção, ou mesmo à luz de um contexto atual (referências atualizadas e baseadas em valores culturais, sociais, políticos e econômicos). Até porque os dados disponíveis, principalmente os advindos das entidades nãogovernamentais (SBN, ABCDT e outras), estão mais afeitos a subsidiar a sobrevivência dos serviços do que para avaliar a efetividade do tratamento em relação ao paciente.

O problema, entretanto, ainda não totalmente respondido à luz do nosso meio, é como realizar estudos de análise de custo-efetividade, dado que não se dispõe de uma base de dados diversificada o suficiente para este fim. Se essa lacuna impede nosso avanço, que nossa inquietação sirva como desafio e recomendação para seu desenvolvimento, por uma política pública de saúde, capaz de desafiar a complexidade em nome da relevância da questão assinalada. 


\section{Resumo}

Este estudo tem por finalidade contribuir com a discussão sobre as possibilidades para a execução de avaliação econômica em saúde, em especial por meio da técnica de custo-efetividade, em relação às terapias renais substitutivas em portadores de falência renal crônica à luz do nosso contexto. Para tanto se realizou uma revisão bibliográfica sobre as intervenções e seus cursos alternativos na perspectiva das proposições metodológicas dispostas na literatura, considerando a disponibilidade de dados e informações no nosso meio que subsidiam este tipo de pesquisa.

Avaliação de Custo-Efetividade; Terapia de Substituição Renal; Falência Renal Crônica

\section{Referências}

1. Drummond MF, Stoddart G, Torrance G. Methods for economic evaluation of health care programmes. New York: Oxford University Press; 1996.

2. Gold MR, Siegel JE, Russel LB, Weinstein MC. Costeffectiveness in health and medicine. New York: Oxford University Press; 1996.

3. Secretaria de Assistência à Saúde, Ministério da Saúde. Estudo epidemiológico brasileiro sobre terapia renal substitutiva: versão preliminar. Brasília: Ministério da Saúde; 2002.

4. Departamento de Informática, Sociedade Brasileira de Nefrologia. Registro brasileiro de diálise, 1997. http://www.epm.br/medicina/registro/97/ rghd97.htm (acessado em Mar/2005).

5. Kuschnir R. Aqui se paga, aqui se faz: pagamento como instrumento de política [Tese de Doutorado]. Rio de Janeiro: Instituto de Medicina Social, Universidade do Estado do Rio de Janeiro; 2004.

6. Romão Jr. JE, Pinto SWL, Canziani ME, Praxedes JN, Santello JL, Moreira JCM. Censo SBN 2002: informações epidemiológicas das unidades de diálise do Brasil. J Bras Nefrol 2003; 25:188-99.

7. Romão Jr. JE. Epidemiologia da doença renal crônica no Brasil 2004: cenário atual. http:// portaweb01.saude.gov.br/saude/visualizar_texto. efm?idtxt_17430 (acessado em Mar/2005)

8. Sesso R. Inquérito epidemiológico em unidades de diálise do Brasil. J Bras Nefrol 2000; 22(3 Suppl 2):23-6.

9. Medronho RA. Epidemiologia. São Paulo: Editora Atheneu; 2003.

\section{Colaboradores}

L. G. Sancho participou da realização da pesquisa e redação do artigo. S. Dain colaborou na revisão do artigo.

10. Mazzuchi N, Carbonell E, Fernandez-Cean J. Importance of blood pressure control in hemodialysis patient survival. Kidney Int 2000; 58:2147-54.

11. Lugon JR, Barros AM, Duarte MEL, Rembold SM, Cruz EAS. Effects of in-center daily hemodialysis upon mineral metabolism and bone disease in end- stage renal disease patients. São Paulo Med J 2001; 119:105-9.

12. Salomão A, Cristelli MP, Santos A, Pereira JE, Gonçalves A, Pessoa GH, et al. Projeto-piloto de hemodiálise curta duração: melhora da qualidade de vida de renais crônicos. J Bras Nefrol 2002; 24:168-75.

13. Jassal SV, Krishna G, Mallick NP, Mendelssohn DC. Attitudes of British Isles nephrologists towards dialysis modality selection: a questionnaire study. Nephrol Dial Transplant 2002; 17:474-7.

14. Nissenson AR, Collins AJ, Dickmeyer J, Litchfield T, Mattern W, McMahill CN, et al. Evaluation of disease-state management of dialysis patients. Am J Kidney Dis 2001; 37:938-44.

15. Klarman HE, Francis JO, Rosenthal GD. Cost-effectiveness analysis applied to the treatment of chronic renal disease. Med Care 1968; 6:48-54.

16. Wit GA, Ramsteijn PG, Charro KT. Economic evaluation of end stage renal disease treatment. Health Policy 1998; 44:215-32. 
17. Clark WF, Churchill DN, Forwell L, Macdonald G, Foster S. To pay or not to pay? A decision and cost utility analysis of angiotensin-coverting-enzyme inhibitor therapy for diabetic nephropothy. CMAJ 2000; 162:195-8.

18. Lee H, Manns B, Taub K, Ghali WA, Dean S, Johnson D, et al. Cost analysis of ongoing care of patients with end-stage renal disease: the impact of dialysis modality and dialysis access. Am J Kidney Dis 2002; 40:611-22.

19. Manns B, Richardson RMA, Donaldson C. To reuse or not reuse? An economic evaluation of hemodialyser reuse versus conventional single-use hemodiyser for chronic hemodialysis. Int J Technol Assess Health Care 2002; 18:81-93.

20. MacLeod A, Daly C, Khan I, Vale L, Campbell M, Wallace S, et al. Comparison of cellulose, modified cellulose and synthetic membranes in the hemodialysis of patients with end-stage real disease. In: The Cochrane Library, Issue 3, 2001. Oxford: Update Software.

21. Mowatt G, Vale L, MacLeod A. Systematic review of effectiveness of home versus hospital or satellite unit hemodialysis for people with end-stage renal failure. Int J Technol Assess Health Care 2004; 20:258-68.

22. Sesso R, Eisenberg M, Stabile C, Draibe S, Ajzen $\mathrm{H}$, Ramos OL. Cost-effectiveness analysis of the treatment of end-stage renal disease in Brazil. Int J Technol Assess Health Care 1990; 6:107-14.

23. Ministério da Saúde. Notas oficiais. http:// portaweb01.saude.gov.br/saude/visualizar_texto. efm?idtxt_13326. (acessado em Mar/2005).

24. Departamento de Informática do SUS. Internações hospitalares do SUS por local de internação - Brasil. http://tabnet.datasus.gov.br/cgi/deftohtm. exe?sih/cnv/rxuf.def (acessado em Out/2007).

25. MacLeod A, Grant A, Donaldson C, Khan I, Campbell M, Daly C. Effectiveness and efficiency of methods of dialysis therapy for end-stage renal disease: systematic reviews. Health Technol Assess 1998; 2:1-166.

26. Mowatt G, Vale L, Perez J, Wyness L, Fraser C, MacLeod A, et al. Systematic review of the effectiveness and cost-effectiveness, and economic evaluation, of home versus hospital or satellite unit haemodialysis for people with end-stage renal failure. Health Technol Assess 2003; 7:1-174.

27. Silva LK. Avaliação tecnológica em saúde: densitometria óssea e terapêuticas alternativas na osteoporose pós-menopausa. Cad Saúde Pública 2003; 19:987-1003.

28. Silva LK. Technology assessment of different levels of neonatal care [Tese de Doutorado]. England: Faculty of Commerce and Social Sciences, University of Birmingham; 1992.

29. Silva LK, Escosteguy CC, Machado CV. Metodologia para a estimativa de padrões de qualidade: o caso do infarto agudo do miocárdio. Cad Saúde Pública 1996; 12 Suppl 2:S71-83.
30. Carvalho MS, Henderson R, Shimakura S, Sousa IPSC. Survival of hemodialysis patients: modelling differences in risk of dialysis centers. Int J Qual Health Care 2003; 15:189-96.

31. Sesso R. Epidemiologia da doença renal crônica no Brasil e sua prevenção. http://www.cve.saude. sp.gov.br/htm/cronicas/irc_prof.htm (acessado em Out/2007).

32. Ciconelli RM, Ferraz MB, Santos W. Tradução para a língua portuguesa e validação do questionário genérico de avaliação de qualidade de vida SF-36 (Brasil SF-36). Rev Bras Reumatol 1998; 39:143-50.

33. Rodrigues-Neto JF, Ferraz MB, Cendoroglo M, Draibe S, Yu L, Sesso R. Quality of life at the initiation of maintenance dialysis treatment: a comparison between the SF-36 and the KDQ questionnaires. Qual Life Res 2000; 9:101-7.

34. Fundação Instituto de Pesquisas Econômicas. Estrutura de custo de serviço de hemodiálise em São Paulo. São Paulo: Fundação Instituto de Pesquisas Econômicas; 1997.

35. Instituto de Saúde Coletiva, Universidade Federal da Bahia. Avaliação econômica do tratamento de terapia renal substitutiva no Estado da Bahia. Salvador: Universidade Federal da Bahia; 2004.

36. Schulpher M, Ades AE, Claxton K. Evidence synthesis, parameter correlation, and probabilistic sensitivity analysis; 2004. http://www.hsrc.ac.uk/ current_research/research_programms/mpes. htm (acessado em Jan/2005).

37. Adam T, Evans D, Koopmanschap M. Cost-effectiveness analysis: can we reduce variability in costing methods? Int J Technol Assess Heath Care 2003; 19:407-20.

38. Kirby L, Vale L. Dialysis for end-stage renal disease. Int J Technol Assess Health Care 2001; 17:181-9.

39. Osta V, Diéguez N, Diéguez S. Evaluación de dos métodos para la determinación de microalbuminuria y de la relación albumina/creatinina en orina. An Pediatr (Barc) 2003; 59:131-7.

40. Castro MSM, Vieira VA, Assunção RM. Padrões espaço-temporais da mortalidade por câncer de pulmão no Sul do Brasil. Rev Bras Epidemiol 2004; 7:131-43.

41. Spiegelhalter DJ, Best N. Bayesian approaches to multiple sources of evidence and uncertainty in complex cost-effectiveness modeling. Cambridge: MRC Biostatistics Unit; 2002.

42. United States Renal Data System. Annual data report. http://www.usrds.org/adr_2002.htm (acessado em Mar/2005).

43. European Renal Association/European Dialysis and Transplant Association. ERA - EDTA Registry annual report 2002. http://www.era-edta-reg.org/ files/annualreports/pdf/AnnRep2002.pdf (acessado em Mar/2005).

44. Sociedade Brasileira de Nefrologia. Censo 2003/2004. http://www.sbn.org.br/Censo/censo 20032004.htm (acessado em Mar/2005).

Recebido em 11/Abr/2006

Versão final reapresentada em 16/Out/2007

Aprovado em 21/Nov/2007 